\title{
New approach to the $k$-independence number of a graph
}

\author{
Yair Caro \\ Dept. of Mathematics and Physics \\ University of Haifa-Oranim \\ Tivon 36006, Israel \\ yacaro@kvgeva.org.il
}

\author{
Adriana Hansberg* \\ Dep. de Matemàtica Aplicada III \\ UPC Barcelona \\ 08034 Barcelona, Spain \\ adriana.hansberg@upc.edu
}

Submitted: Aug 22, 2012; Accepted: Feb 4, 2013; Published: Feb 12, 2013

Mathematics Subject Classifications: 05C69

\begin{abstract}
Let $G=(V, E)$ be a graph and $k \geqslant 0$ an integer. A $k$-independent set $S \subseteq V$ is a set of vertices such that the maximum degree in the graph induced by $S$ is at most $k$. With $\alpha_{k}(G)$ we denote the maximum cardinality of a $k$-independent set of $G$. We prove that, for a graph $G$ on $n$ vertices and average degree $d, \alpha_{k}(G) \geqslant \frac{k+1}{[d \mid+k+1} n$, improving the hitherto best general lower bound due to Caro and Tuza [Improved lower bounds on k-independence, J. Graph Theory 15 (1991), 99-107].
\end{abstract}

Keywords: $k$-independence; average degree

\section{Introduction}

Let $G=(V, E)$ be a graph on $n$ vertices and $k \geqslant 0$ an integer. A $k$-independent set $S \subseteq V$ is a set of vertices such that the maximum degree in the graph induced by $S$ is at most $k$. With $\alpha_{k}(G)$ we denote the maximum cardinality of a $k$-independent set of $G$ and it is called the $k$-independence number of $G$. In particular, $\alpha_{0}(G)=\alpha(G)$ is the usual independence number of $G$. The Caro-Wei bound $\alpha(G) \geqslant \sum_{v \in V} \frac{1}{\operatorname{deg}(v)+1}[11,41]$ is an improvement of the well-known Turán bound for the independent number $\alpha(G) \geqslant \frac{n}{d(G)+1}[40]$, where $d(G)$ is the average degree of $G$. Various results concerning possible improvements and generalizations of the Caro-Wei bound are known (see $[1,2,3,6,10,22,23,25,26,33$, $35,37,38])$. A well known generalization to the $k$-independence number of $r$-uniform

\footnotetext{
* Supported by a Juan de la Cierva Postdoctoral Fellowship; also supported by the Ministry of Education and Science, Spain, and the European Regional Development Fund (ERDF) under project MTM201128800-C02-02; and under the Catalonian Government project 1298 SGR2009
} 
hypergraphs was obtained by Caro and Tuza [12] improving earlier results of Favaron [19] and was extended to non-uniform hypergraphs by Thiele [39]. See also the recent papers $[15,17]$ for updates. An extension of the notion of residue of a graph, notably developed by Fajtlowicz in [18] and Favaron et al. in [20], to the notion of $k$-residue has been developed by Jelen [29]. There has been also much interest in using the Caro-Tuza theorem to algorithmic aspects (see $[24,31,36]$ ). Yet all these lower bounds give asymptotically $\alpha_{k}(G) \geqslant \frac{k+2}{2(d+1)} n$ for $k$ fixed and $d=d(G)$. It is easy to see that in general we cannot hope to get better than $\frac{k+1}{d+1} n$, as can be seen from the graph $G=m K_{d+1}$, consisting of $m$ disjoint copies of the complete graph $K_{d+1}$ and where $d \geqslant k$ and $n=m(d+1)$. So there is still an asymptotic multiplicative gap of a factor of $2 \frac{k+1}{k+2}$. It is worth to mention that there is no known modification of the charming probabilistic proof of the lower bound of Caro-Wei theorem to the situation of $k$-independence that gives a better bound than the Caro-Tuza lower bound. Here, for the sake of being self-contained and to use the same notation, we restate and give the short proof of the Caro-Tuza theorem for graphs. Then we show how to improve this result using further ideas and, in particular, we close the multiplicative gap proving, as a corollary of our main result, that $\alpha_{k}(G) \geqslant \frac{k+1}{\lceil d(G)\rceil+k+1} n$. Doing so, we solve a "folklore" conjecture stated explicitly in [6].

All along this paper, we will use the following notation and definitions. Let $G$ be a graph. By $V(G)$ we denote the set of vertices of $G$ and $n(G)=|V(G)|$ is the order of $G$. $E(G)$ stands for the set of edges of $G$ and $e(G)$ denotes its cardinality. For a vertex $v \in V(G), \operatorname{deg}(v)=\operatorname{deg}_{G}(v)$ is the degree of $v$ in $G$. By $\Delta(G)$ we denote the maximum degree of $G$ and by $d(G)$ the average degree $\frac{1}{n(G)} \sum_{v \in V(G)} \operatorname{deg}(v)$. For a subset $S \subseteq V(G)$, we write $G[S]$ for the graph induced by $S$ in $G$ and $\operatorname{deg}_{S}(v)$ stands for the degree $\operatorname{deg}_{G[S]}(v)$ of $v$ in $G[S]$. Lastly, for a vertex $v \in V(G), G-v$ represents the graph $G$ without vertex $v$ and all the edges incident to $v$ and, for an integer $m \geqslant 1, m G$ is the graph consisting of $m$ disjoint copies of $G$.

The paper is divided into five sections. After this introduction section, we deal in Section 2 with a first naive approach to obtain a lower bound on $\alpha_{k}(G)$ by deleting iteratively vertices of maximum degree until certain point where an old theorem of Lovász [32] is applied. In Section 3, we proceed the same way, taking however a better control on the number of vertices that are deleted and we prove that, for a graph $G$ on $n$ vertices and average degree $d, \alpha_{k}(G) \geqslant \frac{k+1}{[d\rceil+k+1} n$, improving the hitherto best general lower bound due to Caro and Tuza. For this purpose, we define a parameter $f(k, d)$ which approaches from below the best possible ratio $\frac{\alpha(G)}{n(G)}$ for graphs $G$ with $d(G) \leqslant d$, we calculate the exact value of $f(1, d)$ and prove some lower bounds on $f(k, d)$. In Section 4 , we develop some upper bounds on $f(k, d)$. Finally, we present in Section 5 some open problems for further research. 


\section{The naive approach: first improvement}

Let $f_{k}:[0, \infty) \rightarrow \mathbb{R}$ be the function defined by

$$
f_{k}(x)= \begin{cases}1-\frac{x}{2(k+1)}, & \text { if } 0 \leqslant x \leqslant k+1 \\ \frac{k+2}{2(x+1)}, & \text { if } x \geqslant k+1\end{cases}
$$

Observe the following properties of $f_{k}(x)$ :

(P1) $f_{k}(x)$ is a convex function and is strictly monotone decreasing on $[0, \infty)$.

(P2) $f_{k}(i)-f_{k}(i+1) \geqslant f_{k}(j)-f_{k}(j+1)$, for $j \geqslant i \geqslant 0$.

(P3) $i f_{k}(i-1)=(i+1) f_{k}(i)$, for $i \geqslant k+1$.

(P4) $f_{k}(0)=1$ and $f_{k}(k+1)=\frac{1}{2}$.

Theorem 1 (Caro-Tuza for Graphs, [12]). Let $G$ be a graph with degree sequence $d_{1}, d_{2}, \ldots, d_{n}$. Then $\alpha_{k}(G) \geqslant \sum_{i=1}^{n} f_{k}\left(d_{i}\right)$.

Proof. For a subset $X \subseteq V(G)$, define $s(X)=\sum_{x \in X} f_{k}\left(\operatorname{deg}_{X}(x)\right)$. Among all subsets of $X \subseteq V(G)$ such that $s(X)$ is maximum, choose $B$ such that $B$ has the smallest cardinality. In particular, $|B| \geqslant s(B) \geqslant s(V(G))=\sum_{x \in V(G)} f_{k}(\operatorname{deg}(x))$. We will show that $B$ is a $k$-independent set of $\mathrm{G}$. Suppose there is a vertex $y \in B$ such that $\operatorname{deg}_{B}(y)=d \geqslant k+1$. Let $y$ be the vertex of maximum degree in $G[B]$. We will show that $s(B \backslash\{y\}) \geqslant s(B)$, a contradiction to the minimality of $|B|$. For $x \in B \backslash\{y\}$, let $z(x)=1$ if $x y$ is an edge in $G$ and 0 otherwise. Then

$$
\begin{aligned}
s(B \backslash\{y\}) & =\sum_{x \in B \backslash\{y\}} f_{k}\left(\operatorname{deg}_{B \backslash\{y\}}(x)\right)=\sum_{x \in B \backslash\{y\}} f_{k}\left(\operatorname{deg}_{B}(x)-z(x)\right) \\
& =\left(\sum_{x \in B} f_{k}\left(\operatorname{deg}_{B}(x)-z(x)\right)\right)-f_{k}(d) \\
& =s(B)-f_{k}(d)+\sum_{x \in B}\left(f_{k}\left(\operatorname{deg}_{B}(x)-z(x)\right)-f_{k}\left(\operatorname{deg}_{B}(x)\right)\right) \\
& =s(B)-f_{k}(d)+\sum_{x \in B} z(x)\left(f_{k}\left(\operatorname{deg}_{B}(x)-1\right)-f_{k}\left(\operatorname{deg}_{B}(x)\right)\right) \\
& =s(B)-f_{k}(d)+\sum_{x \in B \cap N(y)}\left(f_{k}\left(\operatorname{deg}_{B}(x)-1\right)-f_{k}(\operatorname{deg}(x))\right) .
\end{aligned}
$$

With (P2) we obtain that the last term is at least $s(B)-f_{k}(d)+d\left(f_{k}(d-1)-f_{k}(d)\right)=$ $s(B)-(d+1) f_{k}(d)+d f_{k}(d-1)$ and, since $d f_{k}(d-1)=(d+1) f_{k}(d)$ by $(\mathrm{P} 3)$, this is equal 
to $s(B)$. It follows that $s(B \backslash\{y\}) \geqslant s(B)$, which is a contradiction to the choice of $B$. Hence, $B$ is a $k$-independent set and thus

$$
\alpha_{k}(G) \geqslant|B| \geqslant s(B) \geqslant s(V)=\sum_{x \in V(G)} f_{k}(\operatorname{deg}(x))
$$

Note that, for $k=0$, Theorem 1 yields the Caro-Wei bound. By convexity, the above bound yields also the following corollary.

Corollary 2. For a graph $G$ on $n$ vertices, $\alpha_{k}(G) \geqslant f_{k}(d(G)) n$.

Note that, for $k=0$, Corollary 2 yields the Turán bound $\alpha(G) \geqslant \frac{1}{d(G)+1} n$. Also, if $d(G) \geqslant k+1$, we obtain from this corollary the following one.

Corollary 3. Let $G$ be a graph on $n$ vertices. If $d(G) \geqslant k+1$, then $\alpha_{k}(G) \geqslant \frac{k+2}{2(d(G)+1)} n$.

For a graph $G$, we will denote with $\chi_{k}(G)$ the $k$-chromatic number of $G$, i.e. the minimum number $t$ such that there is a partition $V(G)=V_{1} \cup V_{2} \cup \ldots V_{t}$ of the vertex set $V(G)$ such that $\Delta\left(G\left[V_{i}\right]\right) \leqslant k$ for all $1 \leqslant i \leqslant t$. The following theorem is due to Lovász.

Theorem 4 (Lovász [32], 1966). Let $G$ be a graph with maximum degree $\Delta$. If $k_{1}, k_{2}, \ldots$, $k_{t} \geqslant 0$ are integers such that $\Delta+1=\sum_{i=1}^{t}\left(k_{i}+1\right)$, then there is a partition $V(G)=$ $V_{1} \cup V_{2} \cup \ldots \cup V_{t}$ of the vertex set of $G$ such that $\Delta\left(G\left[V_{i}\right]\right) \leqslant k_{i}$ for $1 \leqslant i \leqslant t$.

Several proofs and generalizations of Lovász's theorem are known. We refer the reader to $[8,9,13,14,34]$. An algorithmic analysis of Lovász theorem with running time $O\left(n^{3}\right)$ is given in [24]. An immediate and well known corollary of Lovász's theorem is Corollary 5, which is useful in the study of defective colorings also known as improper colorings (see $[4,16,21,27])$.

Corollary 5. If $G$ is a graph of maximum degree $\Delta$, then $\chi_{k}(G) \leqslant\left\lceil\frac{\Delta+1}{k+1}\right\rceil$.

Since $\alpha_{k}(G) \geqslant \frac{n}{\chi_{k}(G)}$, the following bound proved in 1986 by Hopkins and Staton follows trivially from the above corollary.

Theorem 6 (Hopkins, Staton [28] 1986). Let $G$ be a graph of order $n$ and maximum degree $\Delta$. Then

$$
\alpha_{k}(G) \geqslant \frac{n}{\left\lceil\frac{\Delta+1}{k+1}\right\rceil} .
$$

The following theorem is a direct consequence of Theorem 6 which generalizes and improves several results concerning relations between $\alpha_{p}(G)$ and $\alpha_{q}(G)$ (see e.g. [5]).

Theorem 7. Let $G$ be a graph and $q \geqslant p \geqslant 0$ two integers. Then $\alpha_{q}(G) \leqslant\left\lceil\frac{q+1}{p+1}\right\rceil \alpha_{p}(G)$. 
Proof. Let $S$ be a maximum $q$-independent set of $G$. Then $\Delta(G[S]) \leqslant q$ and, by Theorem 6 ,

$$
\alpha_{p}(G) \geqslant \alpha_{p}(G[S]) \geqslant \frac{|S|}{\left\lceil\frac{\Delta(G[S])+1}{p+1}\right\rceil} \geqslant \frac{\alpha_{q}(G)}{\left\lceil\frac{q+1}{p+1}\right\rceil},
$$

which implies the statement.

Completing $\Delta+1$ to the next multiple of $k+1$, the following observation is straightforward from Theorem 6 .

Observation 8. Let $G$ be a graph of order $n$ and maximum degree $\Delta$ and let $r$ be an integer such that $0 \leqslant r \leqslant k$ and $\Delta+1+r \equiv 0(\bmod k+1)$. Then

$$
\alpha_{k}(G) \geqslant \frac{k+1}{\Delta+r+1} n
$$

Proof. As clearly $\left\lceil\frac{\Delta+1}{k+1}\right\rceil=\frac{\Delta+r+1}{k+1}$, Theorem 6 implies then $\alpha_{k}(G) \geqslant \frac{k+1}{\Delta+r+1} n$.

When the graph is $d$-regular, we can set $\Delta=d=d(G)$ in Observation 8 and we obtain the following one.

Observation 9. Let $G$ be a d-regular graph on $n$ vertices and let $r$ be an integer such that $0 \leqslant r \leqslant k$ and $d+1+r \equiv 0(\bmod k+1)$. Then $\alpha_{k}(G) \geqslant \frac{k+1}{d+r+1} n$.

So this observation shows that indeed, for $d$-regular graphs, we can close the multiplicative gap of $2 \frac{k+1}{k+2}$ using Lovász's theorem. This serves as an inspiration to trying to close the multiplicative gap in general.

Note that, in practice, the Hopkins-Staton bound can be poor if the maximum degree is far from the average degree. So, our first naive strategy will be to delete a vertex with large degree and, if possible, use induction on the number of vertices. Otherwise, if $\Delta(G)$ is near to the average degree $d(G)$, we will apply Theorem 6 . This is precisely what is done in the next result.

Theorem 10. Let $G$ be a graph on $n$ vertices. Then $\alpha_{k}(G)>\frac{k+1}{d(G)+2 k+2} n$.

Proof. We will proceed by induction on $n$. If $n=1$, the statement is trivial. If $n=2$, $G$ is either $K_{2}$ or $\overline{K_{2}}$. If $G=K_{2}$, then $d(G)=1$ and $\frac{k+1}{d(G)+2 k+2} n=\frac{2(k+1)}{3+2 k}<1 \leqslant \alpha_{k}(G)$ for any $k \geqslant 0$. If $G=\overline{K_{2}}$, then $d(G)=0$ and thus $\frac{k+1}{d(G)+2 k+2} n=1<2=\alpha_{k}(G)$ for all $k \geqslant 0$. Suppose now that $n \geqslant 3$ and that the statement holds for $n-1$. Let $G$ be a graph on $n$ vertices and $v \in V(G)$ a vertex of maximum degree $\Delta$. Define $G^{*}=G-v$. Since any $k$-independent set of $G^{*}$ is also a $k$-independent set of $G, \alpha_{k}(G) \geqslant \alpha_{k}\left(G^{*}\right)$. We distinguish two cases.

Case 1. Suppose that $\Delta \leqslant\lceil d(G)\rceil+k$. Then, by Observation 8, we have

$$
\alpha_{k}(G) \geqslant \frac{k+1}{\Delta+k+1} n \geqslant \frac{k+1}{\lceil d(G)\rceil+2 k+1} n>\frac{k+1}{d(G)+2 k+2} n
$$


and we are done.

Case 2. Suppose that $\Delta \geqslant\lceil d(G)\rceil+k+1$. By induction and with $\Delta \geqslant\lceil d(G)\rceil+k+1 \geqslant$ $d(G)+k+1$, we obtain

$$
\begin{aligned}
\alpha_{k}(G) \geqslant \alpha_{k}\left(G^{*}\right) & >\frac{(k+1)(n-1)}{d\left(G^{*}\right)+2 k+2}=\frac{(k+1)(n-1)}{\frac{2 e\left(G^{*}\right)}{n-1}+2 k+2} \\
& =\frac{(k+1)(n-1)}{\frac{2 e(G)-2 \Delta}{n-1}+2 k+2}=\frac{(k+1)(n-1)}{\frac{d(G) n-2 \Delta}{n-1}+2 k+2} \\
& \geqslant \frac{(k+1)(n-1)}{\frac{d(G) n-2(d(G)+k+1)}{n-1}+2 k+2}=\frac{(k+1) n}{(d(G)+2 k+2) \frac{(n-2) n}{(n-1)^{2}}} \\
& >\frac{k+1}{d(G)+2 k+2} n
\end{aligned}
$$

and the statement follows.

Note that the bound in the previous theorem is better than the Caro-Tuza bound for $k=1$ and $d \geqslant 8$ and for $k \geqslant 2$ and $d \geqslant 2 k+5$. Note also that Theorem 10 already closes the multiplicative factor of $2 \frac{k+1}{k+2}$ for fixed $k$ as $d(G)$ grows. However, to obtain an even better lower bound, we need to get more control on the number of vertices of large degrees that are deleted and to apply Observation 8 in its full accuracy. This will be done in the next section.

We close this section with the following algorithm for obtaining a $k$-independent set of cardinality at least $\frac{k+1}{d(G)+2 k+2} n$ for any graph $G$ on $n$ vertices that yields us the proof of Theorem 10 .

\section{Algorithm 1}

INPUT: a graph $G$ on $n$ vertices and $m$ edges.

(1) Compute $\Delta(G)$ and $d(G)$. GO TO (2).

(2) If $\Delta(G) \leqslant\lceil d(G)\rceil+k$, perform a Lovász partition into $k$-independent sets, choose the largest class $S$ and END. Otherwise choose a vertex $v$ of maximum degree $\Delta(G)$, set $G:=G-v$ and GO TO (1).

\section{OUTPUT: $S$}

The algorithm terminates as, at some step, $\Delta(G) \leqslant\lceil d(G)\rceil+k$ must hold (the latest when $G$ is the empty graph). As already mentioned, the Lovász partition requires a running time of $O\left(n^{3}\right)$, while each other step takes at most $O(n)$ time and the number of iteration steps before performing Lovász partition is at most $n$. Hence, the algorithm runs in at most $O\left(n^{3}\right)$ time. 


\section{Deletions, partitions and a better lower bound on $\alpha_{k}(G)$ - second improvement}

Definition 11. Let $d, k \geqslant 0$ be two integers. We define

$$
f(k, d)=\inf \left\{\frac{\alpha_{k}(G)}{n(G)}: G \text { is a graph with } d(G) \leqslant d\right\} .
$$

Observation 12. Let $d, k \geqslant 0$ be two integers. For every graph $G$ on $n$ vertices and average degree $d(G) \leqslant d, \alpha_{k}(G) \geqslant f(k, d) n$.

The next theorem shows that $f(k, d)$ is convex as a function of $d$.

Theorem 13. Let $d, k, t \geqslant 0$ be integers and $t \leqslant d$. Then $2 f(k, d) \leqslant f(k, d-t)+f(k, d+t)$.

Proof. We will show that for any two graphs $G_{1}$ and $G_{2}$ such that $d\left(G_{1}\right) \leqslant d-t$ and

$d\left(G_{2}\right) \leqslant d+t$, there is a graph $G$ with $d(G) \leqslant d$ such that $2 \frac{\alpha_{k}(G)}{n(G)} \leqslant \frac{\alpha_{k}\left(G_{1}\right)}{n\left(G_{1}\right)}+\frac{\alpha_{k}\left(G_{2}\right)}{n\left(G_{2}\right)}$. Let $G_{1}$ and $G_{2}$ be such graphs and let $n\left(G_{i}\right)=n_{i}$ and $V\left(G_{i}\right)=V_{i}, i=1,2$. Define the graph $G=n_{2} G_{1} \cup n_{1} G_{2}$. Then

$$
\begin{aligned}
2 n_{1} n_{2} d(G)=n(G) d(G) & =n_{2} \sum_{x \in V_{1}} \operatorname{deg}_{G_{1}}(x)+n_{1} \sum_{y \in V_{2}} \operatorname{deg}_{G_{2}}(y) \\
& =n_{2} n_{1} d\left(H_{1}\right)+n_{1} n_{2} d\left(G_{2}\right) \\
& \leqslant n_{2} n_{1}(d-t)+n_{1} n_{2}(d+t)=2 n_{1} n_{2} d
\end{aligned}
$$

implying that $d(G) \leqslant d$ and thus $f(k, d) \leqslant \frac{\alpha_{k}(G)}{n(G)}$. Moreover,

$$
2 f(k, d) \leqslant 2 \frac{\alpha_{k}(G)}{n(G)}=\frac{n_{2} \alpha_{k}\left(G_{1}\right)+n_{1} \alpha_{k}\left(G_{2}\right)}{n_{1} n_{2}}=\frac{\alpha_{k}\left(G_{1}\right)}{n_{1}}+\frac{\alpha_{k}\left(G_{2}\right)}{n_{1}} .
$$

As $G_{1}$ and $G_{2}$ were arbitrarily chosen, it follows that $2 f(k, d) \leqslant f(k, d-t)+f(k, d+t)$.

Before coming to the main theorems of this section, we need the following lemmas.

Lemma 14. Let $d, t \geqslant 0$ be two integers and let $G$ be a graph on $n$ vertices with average degree $d(G) \leqslant d$. Then $G$ has a subgraph $H$ such that either $\Delta(H) \leqslant d+t-1$ and $n(H) \geqslant n-\left\lfloor\frac{n}{d+2 t+1}\right\rfloor$ or $d(H) \leqslant d-1$ and $n(H)=n-\left\lceil\frac{n}{d+2 t+1}\right\rceil$.

Proof. For an $r \geqslant 0$, let $\left\{v_{1}, v_{2}, \ldots, v_{r}\right\}$ be a set of vertices of maximum cardinality such that $\operatorname{deg}_{G_{i+1}}\left(v_{i}\right) \geqslant d+t$, where $G_{i+1}=G_{i}-v_{i}$ and $G_{0}=G$. Suppose first that $r \leqslant\left\lfloor\frac{n}{d+2 t+1}\right\rfloor$ and let $H=G_{r+1}$. Then $H$ has at least $n-r \geqslant n-\left\lfloor\frac{n}{d+2 t+1}\right\rfloor$ vertices and $\Delta(H) \leqslant d+t-1$. Now suppose that $r \geqslant\left\lceil\frac{n}{d+2 t+1}\right\rceil$. Let now $H=G_{q+1}$, where $q=\left\lceil\frac{n}{d+2 t+1}\right\rceil$. Then $n(H)=n-\left\lceil\frac{n}{d+2 t+1}\right\rceil$. Further,

$$
d(H)=\frac{2 e(H)}{n(H)} \leqslant \frac{2(e(G)-(d+t) q)}{n-q} \leqslant \frac{d n-2(d+t) q}{n-q}=\frac{d\left(n-\frac{2(d+t)}{d} q\right)}{n-q} .
$$


Since, for any real numbers $a \geqslant 0$ and $b \geqslant 1$, the function $\frac{a-b x}{a-x}$ is monotonically decreasing in $[0, \infty)$, setting $a=n$ and $b=\frac{2(d+t)}{d}$, we obtain with $q=\left\lceil\frac{n}{d+2 t+1}\right\rceil \geqslant \frac{n}{d+2 t+1}$

$$
\begin{aligned}
d(H) & \leqslant \frac{d\left(n-\frac{2(d+t)}{d} q\right)}{n-q} \leqslant \frac{d\left(n-\frac{2(d+t)}{d} \frac{n}{d+2 t+1}\right)}{n-\frac{n}{d+2 t+1}} \\
& =\frac{d(d+2 t+1)-2(d+t)}{d+2 t}=\frac{d(d+2 t)-(d+2 t)}{d+2 t}=d-1 .
\end{aligned}
$$

Hence, we have shown that $G$ has a subgraph $H$ with either $d(H) \leqslant d-1$ and $n(H)=$ $n-\left\lceil\frac{n}{d+2 t+1}\right\rceil$ or $\Delta(H) \leqslant d+t-1$ and $n(H)=n-\left\lfloor\frac{n}{d+2 t+1}\right\rfloor$.

The following corollary is straightforward from this lemma.

Corollary 15. Let $d, t \geqslant 0$ be two integers. Let $G$ be a graph on $n$ vertices with average degree $d(G) \leqslant d$ and such that $d+2 t+1$ divides $n$. Then $G$ has a subgraph $H$ on $n(H) \geqslant \frac{d+2 t}{d+2 t+1} n$ vertices such that either $d(H) \leqslant d-1$ or $\Delta(H) \leqslant d+t-1$.

Lemma 16. Let $G$ be a graph on $n$ vertices with average degree $d(G) \leqslant d$ and such that $d+2 s+1$ does not divide $n$. Then there is a graph $H$ such that $d+2 s+1$ divides $m=n(H), d(H)=d(G) \leqslant d$ and $\frac{\alpha_{k}(H)}{m}=\frac{\alpha_{k}(G)}{n}$.

Proof. Let $H=(d+2 s+1) G$. Then $m=n(H)=(d+2 s+1) n$ is multiple of $d+2 s+1$, $d(H)=d(G)$ and $\frac{\alpha_{k}(H)}{m}=\frac{(d+2 s+1) \alpha_{k}(G)}{(d+2 s+1) n}=\frac{\alpha_{k}(G)}{n}$.

Let $n$ be an even integer. We denote by $J_{n}$ the graph consisting of a complete graph on $n$ vertices minus a 1 -factor. We are now ready to present the exact value of $f(1, d)$ and the consequences of this result.

Theorem 17. Let $d \geqslant 0$ be an integer. Then the following statements hold.

(1) $f(1, d)= \begin{cases}\frac{2}{d+2}, & \text { if } d \equiv 0(\bmod 2) \\ \frac{2(d+2)}{(d+1)(d+3)}, & \text { if } d \equiv 1(\bmod 2) .\end{cases}$

(2) The equality $f(1, d)=\frac{\alpha_{1}(G)}{n(G)}$ is attained by the graph $G=J_{d+2}$, when $d$ is even, and by $G=(d+3) J_{d+1} \cup(d+1) J_{d+3}$, when $d$ is odd.

(3) $f(1, d) \geqslant \frac{2}{d+2}$.

(4) For every graph $G$ on $n$ vertices, $\alpha_{1}(G) \geqslant \frac{2 n}{\lceil d(G)\rceil+2}$.

Proof. (1) We will prove by induction on $d$ that

$$
f(1, d) \geqslant \begin{cases}\frac{2}{d+2}, & \text { if } d \equiv 0(\bmod 2) \\ \frac{2(d+2)}{(d+1)(d+3)}, & \text { if } d \equiv 1(\bmod 2) .\end{cases}
$$

For $d=0$, clearly $f(1,0)=1=\frac{2}{0+2}$, as the only possible graph $G$ with $d(G) \leqslant 0$ is the empty graph. For $d=1$, let $G$ be a graph with $d(G) \leqslant 1$. Setting $s=1$, we can 
suppose by Lemma 16 that 4 divides $n(G)=n$. Hence, Corollary 15 implies that there is a subgraph $H$ of $G$ on $n(H) \geqslant \frac{3}{4} n$ vertices with $d(H) \leqslant 0$ or $\Delta(H) \leqslant 1$. In both cases we have clearly $\alpha_{1}(G) \geqslant \alpha_{1}(H)=n(H) \geqslant \frac{3}{4} n$ and hence $f(1,1)=\inf \left\{\frac{\alpha_{1}(G)}{n(G)}\right.$ : $G$ graph with $d(G) \leqslant 1\} \geqslant \frac{3}{4}=\frac{2(1+2)}{(1+1)(1+3)}$.

Assume we have proved the statement for $f(1, d-1)$. Now we will prove it for $f(1, d)$, where $d>1$. Let $G$ be a graph on $n$ vertices such that $d(G) \leqslant d$. We distinguish two cases.

Case 1. Suppose that $d \equiv 0(\bmod 2)$. Setting $s=0$, we can suppose by Lemma 16 that $d+1$ divides $n$. By Corollary 15, there is a subgraph $H$ of $G$ on at least $\frac{d}{d+1} n$ vertices with either $d(H) \leqslant d-1$ or $\Delta(H) \leqslant d-1$. Hence, in both cases $d(H) \leqslant d-1$ and thus, by induction, we have

$$
\alpha_{1}(G) \geqslant \alpha_{1}(H) \geqslant f(1, d-1) n(H) \geqslant \frac{2(d+1) d}{d(d+2)(d+1)} n=\frac{2}{d+2} n .
$$

Hence, $f(1, d)=\inf \left\{\frac{\alpha_{1}(G)}{n(G)}: G\right.$ graph with $\left.d(G) \leqslant d\right\} \geqslant \frac{d}{d+2}$ and we are done.

Case 2. Suppose that $d \equiv 1(\bmod 2)$. Set $s=1$. By Lemma 16 , we can suppose that $d+3$ divides $n$. By Corollary 15, there is a subgraph $H$ of $G$ on at least $\frac{d+2}{d+3} n$ vertices with either $d(H) \leqslant d-1$ or $\Delta(H) \leqslant d$. If $d(H) \leqslant d-1$, we can apply the induction hypothesis on $H$ and we obtain

$$
\alpha_{1}(G) \geqslant \alpha_{1}(H) \geqslant f(1, d-1) n(H) \geqslant \frac{2(d+2)}{(d+1)(d+3)} n
$$

and we are done. Suppose finally that $\Delta(H) \leqslant d$. Then, by Theorem 6 and as $d$ is odd, we have

$$
\alpha_{1}(G) \geqslant \alpha_{1}(H) \geqslant \frac{n(H)}{\left\lceil\frac{\Delta(H)+1}{2}\right\rceil} \geqslant \frac{\frac{d+2}{d+3} n}{\left\lceil\frac{d+1}{2}\right\rceil}=\frac{2(d+2)}{(d+1)(d+3)} n .
$$

Thus, in both cases, $f(1, d)=\inf \left\{\frac{\alpha_{1}(G)}{n(G)}: G\right.$ graph with $\left.d(G) \leqslant d\right\} \geqslant \frac{2(d+2)}{(d+1)(d+3)}$ Hence, by induction, the statement holds.

Let $d$ be even. Clearly $\alpha_{1}\left(J_{d+2}\right)=2$ and hence, $f(1, d) \leqslant \frac{\alpha_{1}\left(J_{d+2}\right)}{d+2}=\frac{2}{d+2}$. For $d$ odd, the graph $G=(d+3) J_{d+1} \cup(d+1) J_{d+3}$ has $\alpha_{1}(G)=(d+3) 2+(d+1) 2=4(d+2)$. Hence $f(1, d) \leqslant \frac{\alpha_{1}(G)}{n(G)}=\frac{4(d+2)}{2(d+1)(d+3)}$. Together with the inequalities proven above, it follows

$$
f(1, d)= \begin{cases}\frac{2}{d+2}, & \text { if } d \equiv 0(\bmod 2) \\ \frac{2(d+2)}{(d+1)(d+3)}, & \text { if } d \equiv 1(\bmod 2) .\end{cases}
$$

(2) This follows from the discussion in (1).

(3) It is easily seen that $\frac{2(d+2)}{(d+1)(d+3)} \geqslant \frac{2}{d+2}$. Hence we have always $f(1, d) \geqslant \frac{2}{d+2}$.

(4) From item (3), we obtain $\alpha_{1}(G) \geqslant f(1,\lceil d(G)\rceil) n \geqslant \frac{2}{\lceil d(G)\rceil+2} n$.

We can now state and prove our main result generalizing the proof of Theorem 17 to arbitrary $k$ and $d$. 
Theorem 18. Let $d, k \geqslant 0$ be two integers. Then the following statements hold.

(1) $f(k, d) \geqslant \frac{(k+1)(d+2 t)}{(d+k+t+1)(d+t)} \geqslant \frac{k+1}{d+k+1}$, where $t$ is such that $d \equiv k+1-t(\bmod k+1)$ and $1 \leqslant t \leqslant k+1$.

(2) For $k \geqslant d, f(k, d) \geqslant \frac{2 k+2-d}{2 k+2}$. For $k \geqslant d=1$, the bound is realized by the graph $K_{1, k+1} \cup k K_{1}$ and thus $f(k, 1)=\frac{2 k+1}{2 k+2}$.

(3) For any graph $G$ on $n$ vertices, $\alpha_{k}(G) \geqslant \frac{k+1}{\lceil d(G)\rceil+k+1} n$.

Proof. (1) We will proceed to prove the inequality $f(k, d) \geqslant \frac{(k+1)(d+2 t)}{(d+k+t+1)(d+t)}$ by induction on $d$. If $d=0$, then $d \equiv(k+1)-(k+1)$ and clearly $f(k, 0)=1=\frac{(k+1)(0+2(k+1))}{(0+k+(k+1)+1)(0+(k+1))}$, as the only possible graph $G$ with $d(G) \leqslant 0$ is the empty graph.

Assume $f(k, d-1) \geqslant \frac{(k+1)\left(d-1+2 t^{\prime}\right)}{\left(d+k+t^{\prime}\right)\left(d-1+t^{\prime}\right)}$, where $d-1 \equiv k+1-t^{\prime}(\bmod k+1), 1 \leqslant t^{\prime} \leqslant k+1$, and $d \geqslant 1$. We will prove the statement for $d$. Herefor, we distinguish two cases.

Case 1. Suppose that $d \equiv 0(\bmod k+1)$. Then $t=k+1$. Let $G$ be a graph on $n$ vertices such that $d(G) \leqslant d$. By Lemma 16, setting there $s=0$, we can suppose that $d+1$ divides $n$. Then from Corollary 15 it follows that there is a subgraph $H$ of $G$ on at least $\frac{d}{d+1} n$ vertices such that $d(H) \leqslant d-1$ or $\Delta(H) \leqslant d-1$. In both cases we have $d(H) \leqslant d-1$. Then, as $d-1 \equiv(k+1)-1(\bmod k+1)$, we obtain by induction

$$
\begin{aligned}
\alpha_{k}(G) & \geqslant \alpha_{k}(H) \geqslant \frac{(k+1)(d+1)}{(d+k+1) d} n(H) \geqslant \frac{k+1}{d+k+1} n \\
& =\frac{(k+1)(d+2 t)}{(d+2 t)(d+k+1)} n=\frac{(k+1)(d+2 t)}{(d+k+t+1)(d+t)} n .
\end{aligned}
$$

Thus, $f(k, d)=\inf \left\{\frac{\alpha_{k}(G)}{n(G)}: G\right.$ graph with $\left.d(G) \leqslant d\right\} \geqslant \frac{(k+1)(d+2 t)}{(d+k+t+1)(d+t)}$ and we are done.

Case 2. Suppose that $d \equiv k+1-t(\bmod k+1)$ for some $t$ with $1 \leqslant t \leqslant k$. Using Lemma 16 with $s=t$, we can suppose that $d+2 t+1$ divides $n$. By Corollary 15, there is a subgraph $H$ of $G$ on $n(H) \geqslant \frac{d+2 t}{d+2 t+1} n$ vertices with either $d(H) \leqslant d-1$ or $\Delta(H) \leqslant d+t-1$. If $\Delta(H) \leqslant d+t-1$, then Theorem 6 yields

$$
\begin{aligned}
\alpha_{k}(G) \geqslant \alpha_{k}(H) \geqslant \frac{n(H)}{\left\lceil\frac{\Delta(H)+1}{k+1}\right\rceil} \geqslant \frac{\frac{d+2 t}{d+2 t+1} n}{\left\lceil\frac{d+t}{k+1}\right\rceil} & =\frac{(k+1)(d+2 t)}{(d+2 t+1)(d+t)} n \\
& \geqslant \frac{(k+1)(d+2 t)}{(d+k+t+1)(d+t)} n .
\end{aligned}
$$

Hence, $f(k, d)=\inf \left\{\frac{\alpha_{k}(G)}{n(G)}: G\right.$ graph with $\left.d(G) \leqslant d\right\} \geqslant \frac{(k+1)(d+2 t)}{(d+k+t+1)(d+t)}$ and we are done. Suppose now that $d(H) \leqslant d-1$. Since $d-1 \equiv(k+1)-(t+1)$, we obtain by induction

$$
\begin{aligned}
\alpha_{k}(G) \geqslant \alpha_{k}(H) & \geqslant \frac{(k+1)((d-1)+2(t+1))}{((d-1)+k+(t+1)+1)((d-1)+(t+1))} n(H) \\
& \geqslant \frac{(k+1)(d+2 t+1)}{(d+k+t+1)(d+t)} \cdot \frac{d+2 t}{d+2 t+1} n \\
& =\frac{(k+1)(d+2 t)}{(d+k+t+1)(d+t)} n .
\end{aligned}
$$


Thus, again, $f(k, d) \geqslant \frac{(k+1)(d+2 t)}{(d+k+t+1)(d+k+1)}$ and Case 2 is done.

Hence, by induction, the statement holds. Finally, the inequality $\frac{(k+1)(d+2 t)}{(d+k+t+1)(d+t)} \geqslant \frac{k+1}{d+k+1}$ follows easily.

(2) Let $k \geqslant d$ and let $t$ be such that $d \equiv k+1-t(\bmod k+1)$ and $1 \leqslant t \leqslant k+1$. Then $d=k+1-t$. Hence, with (1),

$$
\begin{aligned}
f(d, k) & \geqslant \frac{(k+1)(d+2 t)}{(d+k+t+1)(d+t)} \\
& =\frac{(k+1)(d+2(k+1-d))}{(d+k+(k+1-d)+1)(d+(k+1-d))}=\frac{2 k+2-d}{2 k+2} .
\end{aligned}
$$

Let $G=K_{1, k+1} \cup k K_{1}$. Then $\alpha_{k}(G)=2 k+1, n(G)=2 k+2$ and $d(G)=\frac{2 k+2}{2 k+2}=1$. Hence, $\frac{2 k+1}{2 k+2} \leqslant f(k, 1) \leqslant \frac{\alpha_{k}(G)}{n(G)}=\frac{2 k+1}{2 k+2}$, obtaining thus equality.

(3) If $G$ is a graph on $n$ vertices, then, using (1), we obtain

$$
\frac{\alpha_{k}(G)}{n} \geqslant f(k,\lceil d(G)\rceil) \geqslant \frac{k+1}{\lceil d(G)\rceil+k+1} .
$$

The proofs of Lemma 14 and Theorem 18 yield us an algorithm for finding, for any graph $G$ on $n$ vertices, a $k$-independent set of cardinality at least $\frac{k+1}{\lceil d(G)\rceil+k+1} n$. It works the following way. It computes $d=d(G)$ and $\Delta(G)$ and finds the integer $t$ such that $0 \leqslant t \leqslant k$ and $d \equiv k+1-t(\bmod k+1)$ (note that the case $t=0$ corresponds here to the case $t=k+1$ of Theorem 18). Then it checks if the graph satisfies the condition $\Delta(G) \leqslant d+t-1$. If so, then it performs a Lovász partition into $k$-independent sets, selects the largest set from it and gives this as output. If not, then a vertex of maximum degree is deleted and the condition on the maximum degree is checked again on the re-

maining graph. This deletion step is repeated up to $\left\lceil\frac{n}{d+2 t+1}\right\rceil$ times, as, by Lemma 14, if the maximum degree is still larger than $d+t-1$, then we are left with a graph with smaller average degree, with which the algorithm starts over again, doing here the inductive step of Theorem 18.

\section{Algorithm 2}

INPUT: a graph $G$ on $n$ vertices and $m$ edges.

(1) Compute $\Delta(G)$ and $d(G)$. Set $d=\lceil d(G)\rceil$ and determine $t$ such that $0 \leqslant t \leqslant k$ and $d \equiv k+1-t(\bmod k+1)$. Set $r:=0$ and GO TO $(2)$.

(2) If $\Delta(G) \leqslant d+t-1$, perform a Lovász partition into $k$-independent sets, choose the largest class $S$ and END. Otherwise GO TO (3).

(3) Set $r:=r+1$. If $r>\left\lceil\frac{n}{d+2 t+1}\right\rceil$, set $n:=n-\left\lceil\frac{n}{d+2 t+1}\right\rceil$ and GO TO (1). Otherwise choose a vertex $v$ of maximum degree $\Delta(G)$, set $G:=G-v$, compute $\Delta(G)$ and GO TO (2).

\section{OUTPUT: $S$}

The algorithm terminates as, at some step, $\Delta(G) \leqslant\lceil d(G)\rceil+t-1$ must hold (the latest when $G$ is the empty graph). Again, the algorithm has a running time of at most $O\left(n^{3}\right)$. 


\section{Upper bounds on $f(k, d)$ and determination of $f(k, d)$ for further small values}

Observe that after Theorems 17(1) and 18(2), we know the exact value of $f(k, d)$ in case $\min \{d, k\} \leqslant 1$. The first pair $(k, d)$ for which an exact value of $f(k, d)$ is not known yet is $(2,2)$. In this section, we develop several upper bounds on $f(k, d)$ as a starting point to future research to obtain further exact values of $f(k, d)$. We will use the following theorem.

Theorem 19 (see [7], p.108). Let $r, g \geqslant 3$ be two integers. If $m$ is an integer with $m \geqslant \frac{(r-1)^{(g-1)}-1}{r-2}$, then there exists an $r$-regular graph of girth at least $g$ and order $2 m$.

Define the function $h(r)=\frac{(r-1)^{r+3}-1}{r-2}$. We will use the particular form of this theorem with $m \geqslant h(r)$, implying that there is an $r$-regular graph of girth at least $r+4$ and order $2 m$.

In the proof of the following theorem, we use the following notation. $\bar{G}$ denotes the complementary graph of $G$. If $F \subseteq E(G)$, then $G-F$ represents the graph $G$ without the edges contained in $F$. For a graph $H$ on at most $n$ vertices, $K_{n}-E(H)$ stands for the complete graph $K_{n}$ without the edges of a subgraph $H$. Further, given two graphs $G$ and $H, G \cup H$ is the graph consisting of one copy of $H$ and one copy of $G$. Finally, the girth of a graph $G$ is denoted by $g(G)$.

Theorem 20. Let $d, k \geqslant 0$ be two integers. Then the following statements hold.

(1) For $d \geqslant k, \frac{k+1}{d+k+1} \leqslant f(k, d) \leqslant \frac{k+1}{d+1}$.

(2) For $d>k, d \equiv 0(\bmod 2)$ and $k \equiv 1(\bmod 2), f(k, d) \leqslant \frac{k+1}{d+2}$.

(3) For $d>k, f(k, d) \leqslant \frac{k+2}{d+3}$.

(4) For $k \geqslant 3, d \geqslant 2 h(k)-k-1$ and $d+k+1 \equiv 0(\bmod 2), f(k, d) \leqslant \frac{k+2}{d+k+1}$.

(5) For $2 \leqslant d \leqslant 4+6 q$, where $q \geqslant 0$ is an integer, $\frac{3}{d+3} \leqslant f(2, d) \leqslant \frac{3}{d+1+\frac{1}{q+1}}$.

(6) For $k \geqslant 2, \frac{k}{k+1} \leqslant f(k, 2) \leqslant \frac{k+1}{k+2+\frac{1}{k+1}}$.

(7) For $k \geqslant 3$, there is a constant $c>0$ auch that $f(k, d)<\frac{k+2}{d+c\left(\frac{d}{2}\right)^{\frac{1}{k+2}}+1}$.

Proof. (1) The lower bound follows from Theorem 18. The upper bound follows from $f(k, d) \leqslant \frac{\alpha_{k}\left(K_{d+1}\right)}{d+1}=\frac{k+1}{d+1}$.

(2) Let $G$ be the graph $K_{d+2}$ minus a 1-factor (this is possible, as $d$ is assumed even). Then $d(G)=d$. Let $T \subseteq V(G)$ be any subset of $k+2$ vertices. As $k+1 \equiv 0(\bmod 2)$, not every vertex of $T$ is covered by the edges of the 1 -factor in $\bar{G}[T]$. Hence, at least one vertex from $T$ is adjacent in $G$ to all other vertices from $T$. Hence, no subset of $k+2$ 
vertices can be a $k$-independent set and thus $\alpha_{k}(G) \leqslant k+1$. This implies $f(k, d) \leqslant \frac{k+1}{d+2}$. (3) Let $d>k$. Consider the graph $G=K_{d+3}-E\left(C_{d+3}\right)$, where $C_{d+3}$ is a cycle of length $d+3$ in $K_{d+3}$. Then $d(G)=d$. Let $T \subseteq V(G)$ a subset of $k+3$ vertices. Since $k+3<d+3=n(G)$, the graph $\bar{G}[T]$ contains no cycles. Hence there is at least one vertex in $v \in V(T)$ which is adjacent in $G[T]$ to all but at most one vertex and hence $\operatorname{deg}_{G[T]}(v) \geqslant k+1$. This implies that $\alpha_{k}(G) \leqslant k+2$ and thus $f(k, d) \leqslant \frac{k+2}{d+3}$.

(4) Let $k \geqslant 3, d \geqslant 2 h(k)-k-1$ and $d+k+1 \equiv 0(\bmod 2)$. By Theorem 19 , there is a $k$-regular graph $H$ with $g(H) \geqslant k+4$ and $n(H)=d+k+1=n$. Consider now the graph $G=K_{n}-E(H)$. Then $d(G)=n-1-k=d$. Let $T \subseteq V(G)$ be a subset of $k+3$ vertices. Since $g(H) \geqslant k+4, \bar{G}[T]$ is a forest. Hence there is at least one vertex in $v \in V(T)$ which is adjacent in $G[T]$ to all but at most one vertex and hence $\operatorname{deg}_{G[T]}(v) \geqslant k+1$. Thus, $\alpha_{k}(G) \leqslant k+2$ and we obtain $f(k, d) \leqslant \frac{k+2}{d+k+1}$.

(5) Consider the graph $G=\left(K_{d+2}-E\left(K_{3}\right)\right) \cup q\left(K_{d+1}-E\left(K_{3}\right)\right)$. Then $n(G)=(q+1) d+q+2$ and $d(G) n(G)=(d-1)(d+1)+3(d-1)+q((d-2) d+3(d-2))=(q+1) d^{2}+(q+3) d-(4+6 q)$. Since $d \leqslant 4+6 q$, it follows that

$$
d(G)=\frac{(q+1) d^{2}+(q+3) d-(4+6 q)}{(q+1) d+q+2} \leqslant \frac{(q+1) d^{2}+(q+3) d-d}{(q+1) d+q+2}=d .
$$

As clearly $\alpha_{2}(G)=3(q+1)$, we obtain therefore, together with Theorem 18 (1),

$$
\frac{3}{d+3} \leqslant f(2, d) \leqslant \frac{3(q+1)}{(q+1) d+q+2}=\frac{3}{d+\frac{q+2}{q+1}}=\frac{3}{d+1+\frac{1}{q+1}} .
$$

(6) Let $k \geqslant 2$ and consider the graph $G=\left(K_{k+3}-E\left(K_{k+1}\right)\right) \cup k K_{1, k+1}$. Then $n(G)=$ $k+3+k(k+2)=k^{2}+3 k+3$ and $d(G) n(G)=2(k+2)+(k+1) 2+2 k(k+1)=2\left(k^{2}+3 k+3\right)=$ $2 n(G)$. Hence, $d(G)=2$. Moreover, it is easy to see that $\alpha_{k}(G)=(k+1)^{2}$. Thus this implies that

$$
f(k, 2) \leqslant \frac{\alpha_{k}(G)}{n(G)}=\frac{(k+1)^{2}}{k^{2}+3 k+3}=\frac{k+1}{k+2+\frac{1}{k+1}} .
$$

Together with the bound from item(2) of Theorem 18, we obtain

$$
\frac{k}{k+1} \leqslant f(k, 2) \leqslant \frac{k+1}{k+2+\frac{1}{k+1}} .
$$

(7) By Theorem 19 there is an $r$-regular graph $H$ with $g(H) \geqslant k+4$ and $n=n(H) \geqslant$ $\frac{2\left((r-1)^{k+3}-1\right)}{r-2}$. Take $n$ even and let $G=K_{n}-E(H)$. Then $d=d(G)=n-1-r$. Let $T \subseteq V(G)$ be a subset of $k+3$ vertices. As $g(H) \geqslant k+4, \bar{G}[T]$ is a forest and thus there is a vertex in $T$ which is adjacent in $G$ to all other vertices from $T$ with the exception of at most one. Hence, $T$ cannot be a $k$-independent set and thus $\alpha_{k}(G) \leqslant k+2$. This implies that $f(k, d) \leqslant \frac{\alpha_{k}(G)}{n} \leqslant \frac{k+2}{d+r+1}$. As $d \sim 2 r^{k+2}$, we have $r \sim\left(\frac{d}{2}\right)^{\frac{1}{k+2}}$, and thus there is a constant $c>0$ such that $r=c\left(\frac{d}{2}\right)^{\frac{1}{k+2}}$, implying that $f(k, d) \leqslant \frac{k+2}{d+c\left(\frac{d}{2}\right)^{k+2}+1}$. 


\section{Open problems}

We close this paper with the following open problems.

Problem 21. Is $f(k, d)$ in fact a minimum for every $k$ and $d$ ? Namely, does

$$
\inf \left\{\frac{\alpha_{k}(G)}{n(G)}: G \text { graph with } d(G) \leqslant d\right\}=\min \left\{\frac{\alpha_{k}(G)}{n(G)}: G \text { graph with } d(G) \leqslant d\right\}
$$

hold?

In case the answer to this problem is positive, this may have several consequences in computing $f(k, d)$.

Problem 22. Is the bound $f(k, d) \geqslant \frac{2 k+2-d}{2 k+2}$ of Theorem $18(2)$ sharp for $k \geqslant d \geqslant 2$ ?

Below are the best possible bounds on $f(2, d)$ we have for $d=0,1, \ldots, 10$.

\begin{tabular}{|c|c|c|c|c|}
\hline$d$ & $\begin{array}{c}\text { lower } \\
\text { bound }\end{array}$ & $\begin{array}{c}\text { upper } \\
\text { bound }\end{array}$ & graph for upper bound & $\begin{array}{c}\text { theorem used } \\
\text { for } \\
\text { upper bound }\end{array}$ \\
\hline 0 & 1 & 1 & $K_{1}$ & - \\
\hline 1 & $5 / 6$ & $5 / 6$ & $K_{1, k+1} \cup K_{1}$ & $18(2)$ \\
\hline 2 & $2 / 3$ & $9 / 13$ & $\left(K_{5}-E\left(K_{3}\right)\right) \cup 2 K_{1,3}$ & $20(6)$ \\
\hline 3 & $1 / 2$ & $3 / 5$ & $K_{5}-E\left(K_{3}\right)$ & $20(5), q=0$ \\
\hline 4 & $4 / 9$ & $1 / 2$ & $K_{6}-E\left(K_{3}\right)$ & $20(5), q=0$ \\
\hline 5 & $7 / 18$ & $6 / 13$ & $\left(K_{7}-E\left(K_{3}\right)\right) \cup\left(K_{6}-E\left(K_{3}\right)\right)$ & $20(5), q=1$ \\
\hline 6 & $1 / 3$ & $2 / 5$ & $\left(K_{8}-E\left(K_{3}\right)\right) \cup\left(K_{7}-E\left(K_{3}\right)\right)$ & $20(5), q=1$ \\
\hline 7 & $11 / 36$ & $6 / 17$ & $\left(K_{9}-E\left(K_{3}\right)\right) \cup\left(K_{8}-E\left(K_{3}\right)\right)$ & $20(5), q=1$ \\
\hline 8 & $5 / 18$ & $6 / 19$ & $\left(K_{10}-E\left(K_{3}\right)\right) \cup\left(K_{9}-E\left(K_{3}\right)\right)$ & $20(5), q=1$ \\
\hline 9 & $1 / 4$ & $2 / 7$ & $\left(K_{11}-E\left(K_{3}\right)\right) \cup\left(K_{10}-E\left(K_{3}\right)\right)$ & $20(5), q=1$ \\
\hline 10 & $7 / 30$ & $6 / 23$ & $\left(K_{12}-E\left(K_{3}\right)\right) \cup\left(K_{11}-E\left(K_{3}\right)\right)$ & $20(5), q=1$ \\
\hline
\end{tabular}

*Lower bounds are from Thm. 18(2) in case $d=1$ and Thm. 18(1) else.

Problem 23. Improve upon the values given in the table.

In order to better understand $f(k, d)$, we can define

$$
f(k, d, \Delta)=\inf \left\{\frac{\alpha_{k}(G)}{n(G)}: G \text { is a graph with } d(G) \leqslant d \text { and } \Delta(G) \leqslant \Delta\right\},
$$

where $\Delta \geqslant d$, and $k$ are all nonnegative integers. Observe that $f(k, d)=\inf \{f(k, d, \Delta): \Delta \geqslant d\}$ and hence a knowledge on $f(k, d, \Delta)$ may help in obtaining better bounds on $f(k, d)$. For instance, let us take $f(2,2,3)$. Observe that, from Theorem $18(2), f(2,2,3) \geqslant f(2,2) \geqslant \frac{2}{3}$. Further, consider the graph $G=R_{8} \cup 4 K_{1,3}$ on 24 vertices, where $R_{8}$ is the graph depicted below (note that $R_{8}$ is the extremal graph for Reed's upper bound of $\frac{3}{8} n$ on the domination 


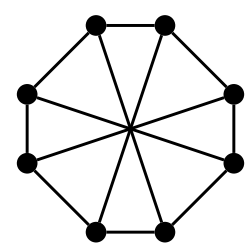

The graph $R_{8}$.

number for graphs on $n$ vertices with minimum degree at least 3 ), and observe that $\alpha_{2}(G)=17$, $n(G)=24$ and $\Delta(G)=3$. Then, it follows that $\frac{2}{3} \leqslant f(2,2) \leqslant f(2,2,3) \leqslant \frac{17}{24}$.

But if we consider for instance the graph $H=\left(K_{5}-E\left(K_{3}\right)\right) \cup 2 K_{1,3}$, then we have there $\alpha_{2}(H)=9, n(H)=13$ and $\Delta(H)=4$ and thus $\frac{2}{3} \leqslant f(2,2) \leqslant f(2,2,4) \leqslant \frac{9}{13}$, which is better than the bound $\frac{17}{24}$ obtained with the graph $G$. Thus, we would like to state the following question.

Problem 24. Obtain lower and upper bounds on $f(k, d, \Delta)$.

\section{References}

[1] M. Ajtai, J. Komlós, and E. Szemerédi. A note on Ramsey numbers. J. Comb. Theory Ser. A, 29:354-360, 1980.

[2] N. Alon, J. Kahn, P. D. Seymour. Large induced degenerate subgraphs. Graphs Combin. 3(3):203-211, 1987.

[3] N. Alon and J.H. Spencer. The probabilistic method (2nd ed.). Wiley, 2000.

[4] J.-C. Bermond, F. Havet, F. Huc, C. Linhares Sales Improper coloring of weighted grid and hexagonal graphs. Discrete Math. Algorithms Appl. 2(3):395-411, 2010.

[5] M. Blidia, M. Chellali, O. Favaron, N. Meddah. Maximal $k$-independent sets in graphs. Discuss. Math. Graph Theory 28(1):151-163, 2008.

[6] A. Bojilov, Y. Caro, A. Hansberg, N. Nenov. Partitions of graphs into small and large sets. submitted.

[7] B. Bollobás. Extremal graph theory, Reprint of the 1978 original. Dover Publications, Inc., Mineola, NY, xx+488 pp, 2004.

[8] B. Bollobás, B. Manvel. Optimal vertex partitions. Bull. London Math. Soc.11(2):113-116, 1979 .

[9] O. V. Borodin, A. V. Kostochka, B. Toft. Variable degeneracy: extensions of Brooks' and Gallai's theorems. Discrete Math. 214(1-3):101-112, 2000.

[10] P. Borowiecki, F. Göring, J. Harant, D. Rautenbach. The potential of greed for independence. J. Graph Theory 71(3):245-259, 2012.

[11] Y. Caro. New results on the independence number. Tech. Report, Tel-Aviv University, 1979.

[12] Y. Caro, Z. Tuza. Improved lower bounds on k-independence. J. Graph Theory 15(1):99$107,1991$. 
[13] P. A. Catlin. Another bound on the chromatic number of a graph Discrete Math. 24(1):1-6, 1978.

[14] P. A. Catlin. Graph decompositions satisfying extremal degree constraints J. Graph Theory 2(2):165-170, 1978.

[15] M. Chellali, O. Favaron, A. Hansberg, L. Volkmann. $k$-domination and $k$-independence in graphs: a survey. Graphs Combin. 28(1):1-55, 2012.

[16] L. J. Cowen, R. H. Cowen, D. R. Woodall. Defective colorings of graphs in surfaces: partitions into subgraphs of bounded valency. J. Graph Theory 10(2):187-195, 1986.

[17] B. Csaba, T. A. Plick, A. Shokoufandeh. A note on the Caro-Tuza bound on the independence number of uniform hypergraphs. Australas. J. Combin. 52, 235-242, 2012.

[18] S. Fajtlowicz On conjectures of Graffiti, III. Congr. Numer. 66:23-32, 1988.

[19] O. Favaron. k-domination and k-independence in graphs. Ars Combin. 25(C):159-167, 1988.

[20] O. Favaron, M. Mahéo, J.F. Saclé. On the residue of a graph. J. Graph Theory 15:39-64, 1991.

[21] M. Frick, M. Henning. Extremal results on defective colorings of graphs. Discrete Math. 126(1-3):151-158, 1994.

[22] F. Göring, J. Harant, D. Rautenbach and I. Schiermeyer. On $\mathcal{F}$-independence in graphs. Discuss. Math. Graph Theory 29(2):377-383, 2009.

[23] J. R. Griggs. Lower Bounds on the Independence Number in Terms of the Degrees. Journal of Combinatorial Theory, Series B 34(1):22-39, 1983.

[24] M. M. Halldórsson and H. Ch. Lau. Low-degree Graph Partitioning via Local Search with Applications to Constraint Satisfaction, Max Cut, and Coloring. Journal of Graph Algorithms and Applications 1(3):49-61, 1997.

[25] J. Harant, D. Rautenbach. Independence in connected graphs Discrete Appl. Math. 159(1):79-86, 2011.

[26] J. Harant and I. Schiermeyer. On the independence number of a graph in terms of order and size. Discrete Math. 232:131-138, 2001.

[27] F. Havet, R. J. Kang, J.-S. Sereni. Improper coloring of unit disk graphs. Networks 54(3):150-164, 2009.

[28] G. Hopkins and W. Staton. Vertex partition and $k$-small subsets of graphs. Ars Combin. 22:19-24, 1986.

[29] F. Jelen. $k$-Independence and the $k$-residue of a graph. J. Graph Theory 32:241-249, 1999.

[30] Y. Li, W. Zang. Differential methods for finding independent sets in hypergraphs. SIAM J. Discrete Math. 20(1):96-104, 2006.

[31] E. Losievskaja. Approximation algorithms for independent set problems on hypergraphs. Ph.D. thesis, Reykjavík University, 2009, http://hdl.handle.net/1946/7410.

[32] L. Lovász. On decompositions of graphs. Studia Sci. Math Hungar. 1:237-238, 1966.

[33] O. Murphy. Lower bounds on the stability number of graphs computed in terms of degrees. Discrete Math. 90(2):201-211, 1991. 
[34] L. Rabern. Partitioning and coloring with degree constraints. arXiv:1202.5855v1.

[35] S.M. Selkow. The independence number of a graph in terms of degrees. Discrete Math. 132:363-365, 1994.

[36] H. Shachnai, A. Srinivasan. Finding large independent sets in graphs and hypergraphs. SIAM J. Discrete Math. 18(3):488-500 (electronic), 2004/05.

[37] J.B. Shearer. A note on the independence number of triangle-free graphs. Discrete Math. 46:83-87, 1983.

[38] J.B. Shearer. A note on the independence number of triangle-free graphs. II. J. Comb. Theory, Ser. B 53:300-307, 1991.

[39] T. Thiele. A lower bound on the independence number of arbitrary hypergraphs. J. Graph Theory 30(3):213-221, 1999.

[40] P. Turán. An extremal problem in graph theory (hungarian). Mat Fiz Lapok 48:436-452, 1941.

[41] V. K. Wei. A lower bound on the stability number of a simple graph. Bell Laboratories Technical Memorandum, 81-11217-9, Murray Hill, NJ, 1981. 